\title{
PEDOT:PSS/Graphene Nanocomposite Hole-Injection Layer in Polymer Light-Emitting Diodes
}

\author{
Chun-Hsuan Lin, ${ }^{1}$ Kun-Tso Chen, ${ }^{1}$ Jeng-Rong Ho, ${ }^{2}$ \\ J.-W. John Cheng, ${ }^{1}$ and Raymond Chien-Chao Tsiang ${ }^{3}$ \\ ${ }^{1}$ Department of Mechanical Engineering, National Chung Cheng University, Chiayi 621, Taiwan \\ ${ }^{2}$ Department of Mechanical Engineering, National Central University, Taoyuan 320, Taiwan \\ ${ }^{3}$ Department of Chemical Engineering, National Chung Cheng University, Chiayi 621, Taiwan
}

Correspondence should be addressed to Jeng-Rong Ho, jrho@ncu.edu.tw

Received 15 July 2011; Accepted 4 January 2012

Academic Editor: Junfeng Geng

Copyright (C) 2012 Chun-Hsuan Lin et al. This is an open access article distributed under the Creative Commons Attribution License, which permits unrestricted use, distribution, and reproduction in any medium, provided the original work is properly cited.

\begin{abstract}
We report on effects of doping graphene in poly(3,4-ethylenedioxythiophene): poly(styrene sulfonate), PEDOT:PSS, as a PEDOT:PSS/graphene nanocomposite hole injection layer on the performance enhancement of polymer light-emitting diodes (PLEDs). Graphene oxides were first synthesized and then mixed in the PEDOT:PSS solution with specifically various amounts. Graphenes were reduced in the PEDOT:PSS matrix through thermal reduction. PLED devices with hole-injection nanocomposite layer containing particular doping concentration were fabricated, and the influence of doping concentration on device performance was examined by systematically characterizations of various device properties. Through the graphene doping, the resistance in the hole-injection layer and the turn-on voltage could be effectively reduced that benefited the injection and transport of holes and resulted in a higher overall efficiency. The conductivity of the hole-injection layer was monotonically increased with the increase of doping concentration, performance indices from various aspects, however, did not show the same dependence because faster injected holes might alter not only the balance of holes and electrons but also their combination locations in the light-emitting layer. Results show that optimal doping concentration was the case with $0.03 \mathrm{wt} \%$ of graphene oxide.
\end{abstract}

\section{Introduction}

Polymer light emitting diodes (PLEDs), or more generally organic light emitting diodes (OLEDs), have been investigated in both commercial and academic fields because of their unique advantages such as light weight, thin structure, high resolution, and low driving voltage. The fundamental structure of a PLED consists of multiple organic thin films sandwiched between two electrodes. Thus, the device performance can be enhanced through improving characteristics of the thin films. Recently, due to their distinctive properties, the applications of nanomaterials and nanostructures to performance enhancement of PLEDs have been keeping attracting much research attention.

The literature shows, by doping nanoparticles (NPs) of $\mathrm{TiO}_{2}$ and $\mathrm{SiO}_{2}$ into the emitting layer MEH-PPV (poly(2-methoxy-5(2'-ethyl)hexoxy-phenylenevinylene)) in PLEDs, that the device characteristics in current density, and luminance were obviously improved $[1,2]$. This was because the doped NPs enhanced the abilities of charge injection and charge transport. In addition to their effectiveness in the light-emitting layer, the effect of $\mathrm{TiO}_{2}$ NPs in the hole transport layer, PEDOT:PSS (poly(3,4ethylenedioxythiophene):poly(styrene sulfonate)), was also studied. The $\mathrm{TiO}_{2}$ NPs were again demonstrated to have ability to enhance injection of holes; consequently the turn-on voltage of the device was reduced. The modification in hole injection can also be accomplished by introducing an additional SWNTs/PVK (single carbon nanotubes/polyvinylcarbazole) nanocomposite layer between the original hole transport layer PEDOT:PSS and lightemitting layer $\mathrm{Alq}_{3}[3]$.

In addition to playing a positive role in both the lightemitting and hole transport layers, the use of NPs and nanostructures to enhance electron injection was also widely reported. By introducing $\mathrm{ZnO}$ NPs between the cathode and 


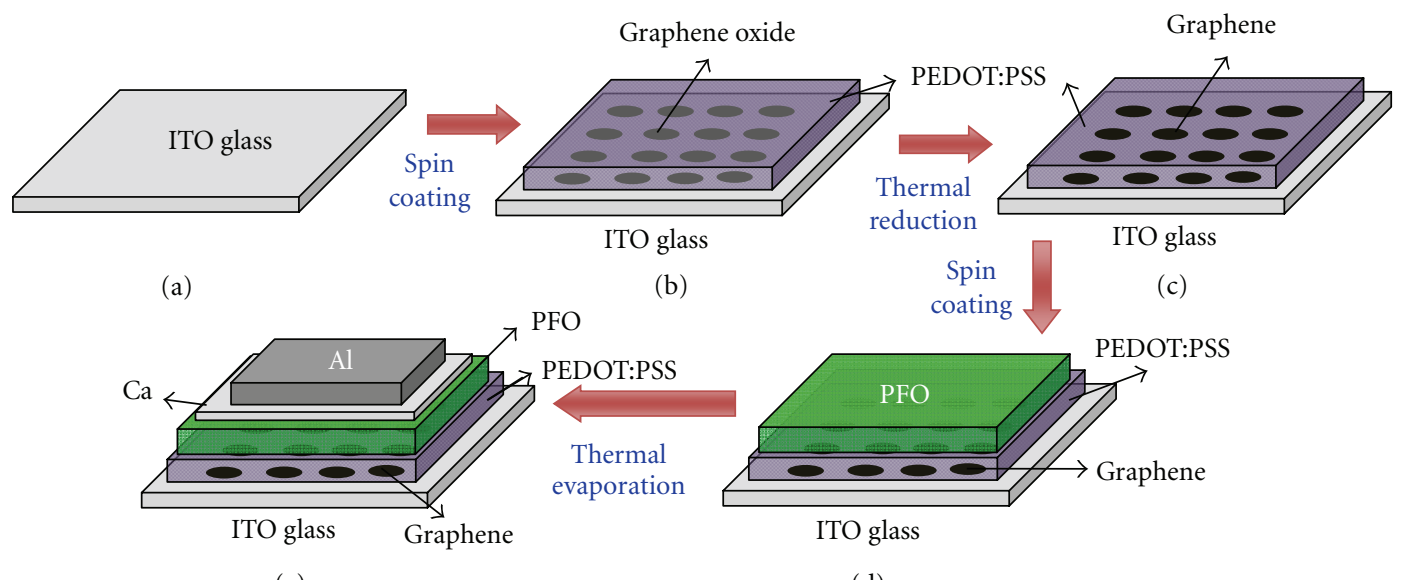

(e)

(d)

FIgURE 1: Schematics illustrating the fabrication steps of a PLED device.

the emitting layers, the device performance was enhanced because the $\mathrm{ZnO}$ NPs helped the injection of electrons and blocked the transport of holes to the cathode [4]. The employment of SWNTs/MEH-PPV nanocomposites as the electron transport layer showed high conductive ability in electrons, thereby enhancing the device efficiency [5]. The addition of $\mathrm{SiO}_{2} \mathrm{NPs}$ and $\mathrm{SiO}_{2} / \mathrm{TiO}_{2} \mathrm{NP}$ blends between the cathode and the emitting layer as an electron injection layer was also studied and proved to be able to advance the electron injection and the internal quantum efficiency [6]. The optoelectronic efficiency of OLEDs can thus be practically improved by the measures of introducing appropriate NPs or nanostructures in and/or between organic thin films.

Indium tin oxide (ITO) is widely used as the anode in OLEDs due to its high electrical conductivity and high transparency. The performance of OLEDs is, of course, affected by the ability of injection of holes from the ITO. Thus, modification of ITO anode, such as introducing an additional hole injection layer, is an important research issue. Different hole injection layers, such as $\mathrm{SiO}_{2}$ [7], $\mathrm{NiO}$ [8], carbon [9], Pt [10], PANI (polyaniline) [11], PEDOT:PSS $[12,13]$, and so forth, have been inserted between the ITO anode and the hole transport layer in order to decrease the injection barrier. Among them, PEDOT:PSS is widely used because of its high conductivity, high transmittance, and good film-formation properties.

By doping with suitable inorganic nanomaterials, the electrical properties of PEDOT:PSS can be improved and the resulting PEDOT:PSS nanocomposite layer in PLEDs is expected to facilitate the injection of holes. Carbon nanotubes (CNTs) exhibit high work function, high conductivity, and have low absorption coefficient. Therefore, the CNT is a good candidate of dopants for PEDOT:PSS. In the literature [14-16], the PEDOT:PSS/CNT nanocomposite was indeed shown to be an effective hole-injection layer. With the existence of CNTs, the turn-on voltage was reduced and the luminance intensity as well as the device's overall efficiency was enhanced.

Graphene, a two-dimensional structure consisting of $\mathrm{sp}^{2}$ hybridized carbon, has been depicted with excellent electrical, mechanical, thermal, chemical, and optical properties.
Therefore, its application potential in the future electronics and optoelectronics keeps attracting very much research attention. Recently, it has been reported that graphene could be doped with MEH-PPV to act as acceptor in the luminous layer [17] and inserted as a hole injection layer in a PLED [18]. Hole injection improvement through blending new synthesized solution-processable graphene oxides with PEDOT:PSS is also feasible $[19,20]$. In this study, we investigate the effect of PEDOT:PSS/graphene nanocomposite hole-injection layer on the performance enhancement of PLEDs. The influence of doping concentration on device performance was systematically studied from various aspects of characteristics. Based on the results obtained, variations of properties with doping concentration were presented, and the mechanisms corresponding to these variations were discussed. The optimal doping concentration was also reported.

\section{Experiments}

In this study, the effect of graphene, being doped into PEDOT:PSS as a PEDOT:PSS/graphene nanocomposite layer for hole injection, on the performance enhancement of a PLED device is examined. The employed PEDOT:PSS solution was commercially available, Clevios PVP AI4083. The graphene was obtained by thermal reduction from graphene oxide (GO) which was synthesized using the Hummers method [21]. The synthesized GO powder was then dropped into the PEDOT:PSS solution to form the initial PEDOT:PSS/GO solution for the subsequent spincoating process. According to the amount (weight) of the GO powder mixed in the PEDOT:PSS solution, five resulting PEDOT:PSS/GO solutions with different weight percentages, the weight ratio of GO powder and PEDOT:PSS/GO solution, were prepared to investigate the effect of graphene content on the device's performance. They were $0.005,0.01$, $0.03,0.05$, and 0.1 weight percentages (wt $\%)$, respectively.

The steps of fabricating a PLED device are schematically demonstrated in Figure 1. Glass with precoated indium tin oxide (ITO) thin film was used as the substrate, Figure 1(a). The PEDOT:PSS/GO solution with a particular $\mathrm{wt} \%$ of GO was then spin coated on the ITO substrate at 


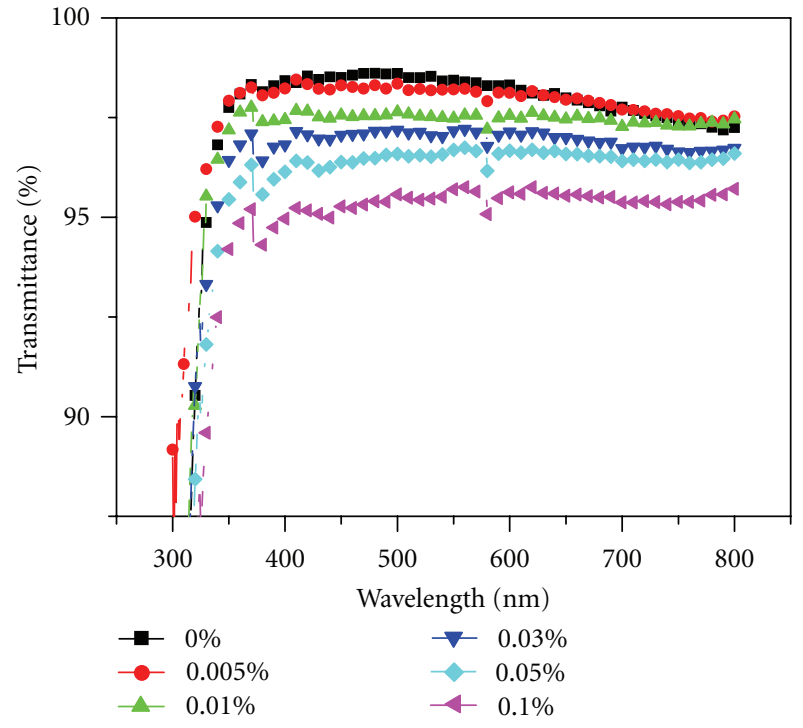

Figure 2: Measured curves showing transmittance as a function wavelength for PEDOT:PSS/graphene layers with various graphene concentrations.

$7000 \mathrm{rpm}$ for $60 \mathrm{~s}$, Figure 1(b). All samples of the resulting PEDOT:PSS/graphene nanocomposite layer with specific content of graphene were obtained by immediately putting the PEDOT:PSS/GO liquid films, coated on the ITO substrates, in an oven and underwent a thermal treatment at $260^{\circ} \mathrm{C}$ for 1 hour in order to reduce the graphene oxide into graphene, Figure 1(c). To avoid thermal damage of PEDOT:PSS, here the treatment temperature is not high and GO might not be reduced completely [22]. The emitting layer was polyfluorene (PFO). It was prepared in chloroform with $0.7 \mathrm{wt} \%$ in concentration and coated upon the PEDOT:PSS/graphene nanocomposite by spin coating at $3000 \mathrm{rpm}$ for $60 \mathrm{~s}$ and followed by baking at $70^{\circ} \mathrm{C}$ for $5 \mathrm{~min}$ on a hot plate to remove any residual solvent, Figure 1(d). Finally, the cathode metals, a bilayered structure of Ca $(10 \mathrm{~nm})$ and $\mathrm{Al}(100 \mathrm{~nm})$, were deposited on the PFO using thermal evaporation at a vacuum pressure of $6 \times 10^{-6}$ torr, Figure 1(e).

The transmittance measurement was executed using an UV/vis spectrometer (Thermo Helios Omega). The device's $J-V$ curve (current density, ampere per unit square meter, as a function of voltage) and $L-V$ curve (luminescence, candela per square meter, as a function of voltage) were characterized using a source meter (Keithley 2400) and a spot luminance meter (LS-100), respectively. The measurement of ionization potential (HOMO, highest occupied molecular orbital) and the electron affinity (LUMO, lowest unoccupied molecular orbital) of the PEDOT:PSS/graphene composite thin film was executed using a cyclic voltammetry $(\mathrm{CV}, \mathrm{CH}$ Instruments-611C) $[23,24]$.

\section{Results and Discussion}

To understand the effect of the existence of graphene on PLED's light transmission, the transmittance, over the

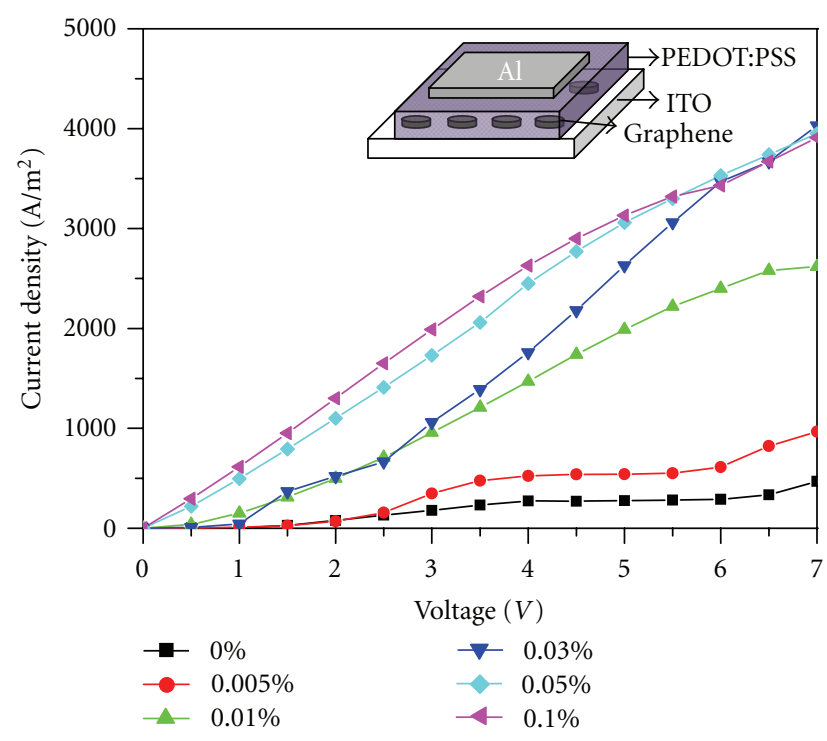

Figure 3: The $J-V$ characteristics of hole-only device at various graphene concentrations. The inset shows the device configuration.

spectrum from 300 to $800 \mathrm{~nm}$, of the PEDOT:PSS/graphene nanocomposite thin film was first examined. Figure 2 shows that the transmittances of all the studied nanocomposites were smaller than that of the pure PEDOT:PSS thin film $(0 \%)$ and were decreased with the increase of the graphene concentration. However, even for the nanocomposite film with the highest graphene concentration $(0.1 \%)$, the transmittance within the measured spectrum range was still higher than $95 \%$. This indicates that the existence of graphene in the PEDOT:PSS did not significantly affect the luminance output of the PLEDs.

The influence of graphene on the $J-V$ characteristics was solely examined using a hole-only device. The device configuration is schematically shown in the inset of Figure 3 and the fabrication steps for the nanocomposite layer, and the metal electrode layer followed the same procedures as those in the PLED device and were described in Section 2. The $J-V$ characteristics for the hole-only devices with nanocomposite layers having various graphene concentrations are shown in Figure 3 . For the case of $0.005 \%$, the $J-V$ curve was close to that for the undoped case. However, the enhancement of current density at all voltages was still observable. With the increase of the doping concentration to $0.01 \%$, the enhancement of current density at all voltages became more obvious. This trend remained as the doping concentration was further enhanced. However, as the concentration was augmented from 0.05 to $0.10 \%$, the degree of enhancement in current density again became less evident. These results indicate that, with the doping of graphene, the conductivity in the PEDOT:PSS thin film could be markedly improved and the more the doped concentration the higher the conductivity. However, when the doping concentration was high enough, the rate of augmentation in current density owing to the succeeding increase of doping concentration became less apparent. 


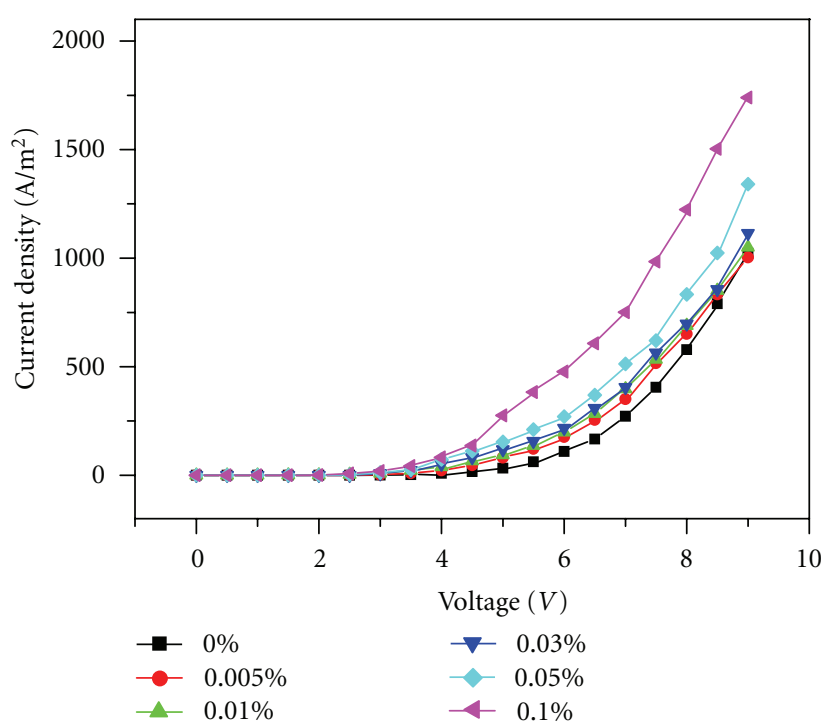

(a)

FIgURE 4: The effect of graphene concentration on the $J-V$ curve nanocomposite as the hole-injection layer.

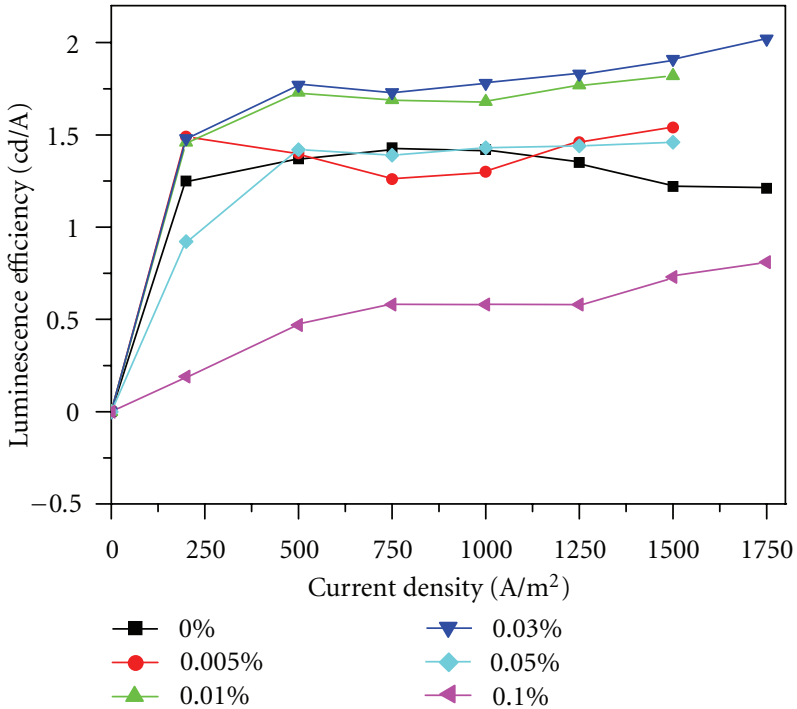

(a)

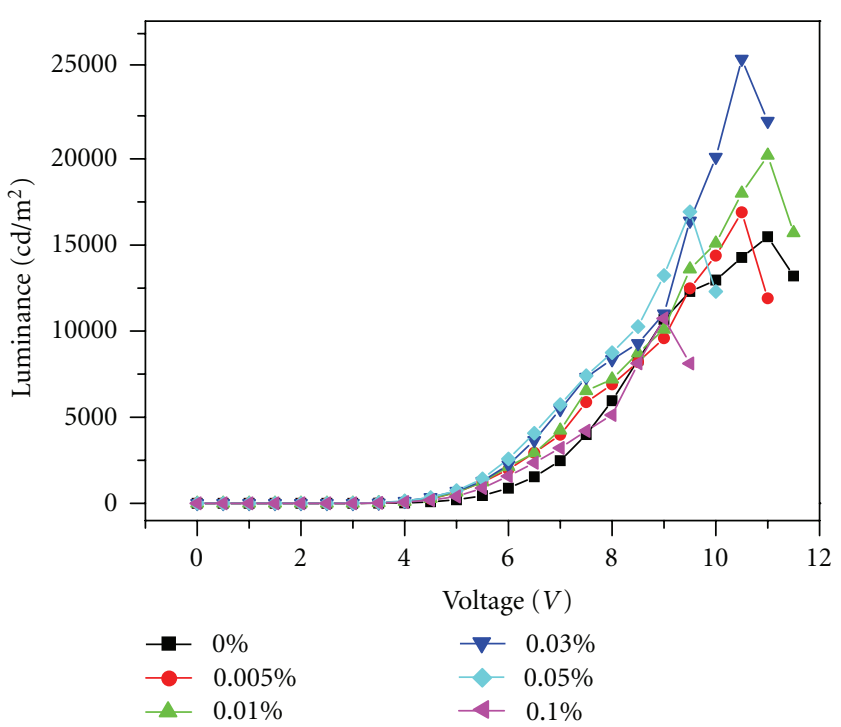

(b)

(a) and $L-V$ curves (b) of PLED using PEDOT:PSS/graphene

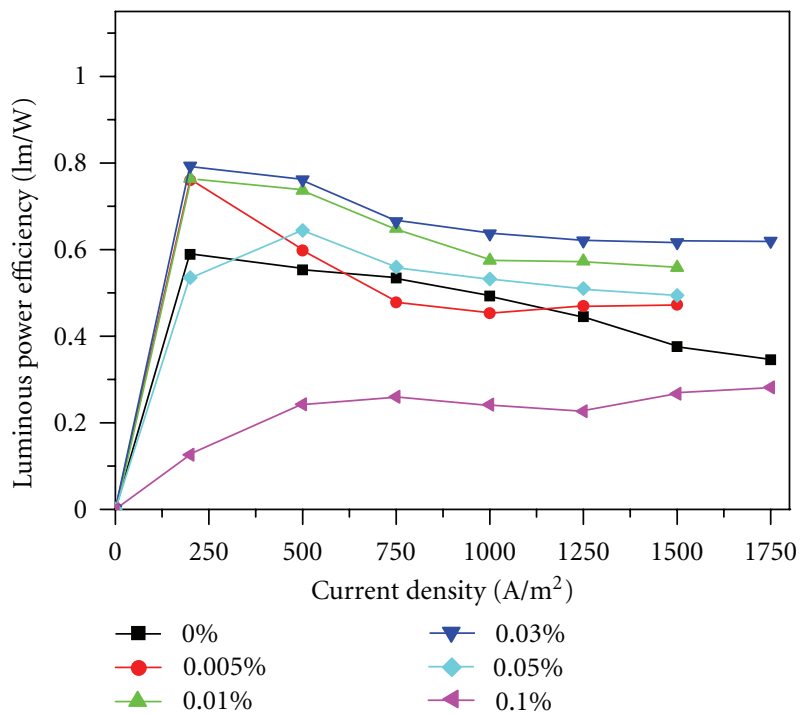

(b)

FIgURE 5: The effect of graphene concentration on luminous efficiency (a) and luminous power efficiency (b) of PLEDs using PEDOT:PSS/graphene nanocomposite as the hole-injection layer.

Figures 4(a) and 4(b) show that the characteristics of $J$ $V$ and $L-V$ of the PLED devices, respectively. In Figure 4(a), the curve for the pure PEDOT:PSS showed the lowest current density and, on the other hand, the device with the highest doping concentration of graphene, $0.1 \%$, had the highest current density. The curves in this figure clearly exhibited the tendency that the current density increased with the increase of doping concentration. With the increase of graphene content, the resistance of the composite films was reduced that resulted in decrease of the device's bulk resistance and led to an increase in current density. Figure 4(b) shows the luminance of a PLED device was enhanced monotonically with the increase of voltage and the device broke down when the voltage was too large. The reachable maximum luminance for the device using the PEDOT:PSS as the holeinjection layer was about $15480 \mathrm{~cd} / \mathrm{m}^{2}$ occurring at the voltage of $11 \mathrm{~V}$ and that could be increased to $25800 \mathrm{~cd} / \mathrm{m}^{2}$ at even a little bit lower voltage of $10.5 \mathrm{~V}$ for the device with doping concentration of $0.03 \%$.

Because of different doping concentrations and unavoidable uncertainties in sample preparation and device fabrication, the reachable maximum luminance for each device would occur at different voltages. Other often-used indices for evaluating performance of a PLED device include the luminous efficiency and the luminous power efficiency. These two indices for the currently studied devices are presented in Figure 5. Figures 5(a) and 5(b), respectively, show the luminous efficiency $(\mathrm{cd} / \mathrm{A}$, candela per unit 
TABLE 1: Electrical characteristics of the PLEDs with various concentrations of PEDOT:PSS/graphene composite layers.

\begin{tabular}{lcccccc}
\hline \multirow{2}{*}{ Device characteristics } & \multicolumn{5}{c}{ Weight percentage of graphene oxide (wt\%) } \\
& 0 & 0.005 & 0.01 & 0.03 & 0.05 \\
\hline$V_{T}(\mathrm{~V})$ & 3.12 & 3 & 2.8 & 2.75 & 2.6 & 2.75 \\
Max. luminance $\left(\mathrm{cd} / \mathrm{m}^{2}\right)$ & 15480 & 16900 & 20200 & 25800 & 16930 \\
Max. current eff. $(\mathrm{cd} / \mathrm{A})$ & 1.43 & 1.56 & 1.88 & 2.09 & 1.48 & 0.1 \\
Max. power eff. $(\mathrm{lm} / \mathrm{W})$ & 0.58 & 0.76 & 0.76 & 0.79 & 0.64 \\
\hline
\end{tabular}

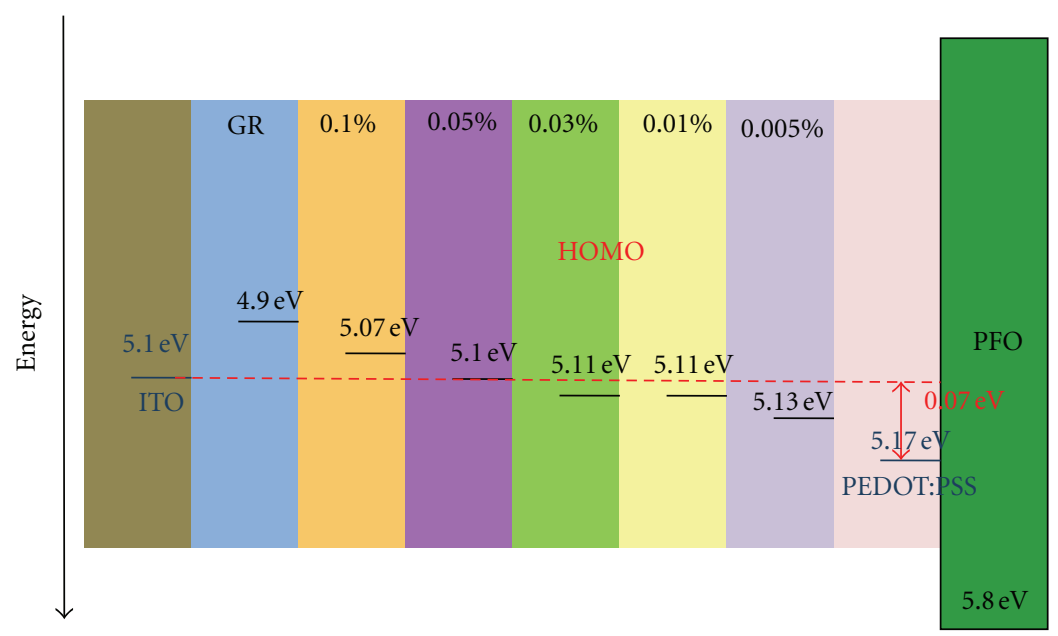

FIGURE 6: Schematic energy level diagram for the PEDOT:PSS/graphene nanocomposites with different graphene concentrations.

current) and luminous power efficiency ( $\mathrm{lm} / \mathrm{W}$, luminance per unit power) as a function of current density. For the undoped device, the luminous efficiency and luminous power efficiency were $1.43 \mathrm{~cd} / \mathrm{A}$ and $0.58 \mathrm{~lm} / \mathrm{W}$, respectively. With introducing graphene into the PEDOT:PSS film, both efficiencies could be improved. The results show that both efficiencies were augmented with the increase of doping concentration when the doping concentration was low. Their dependent relationship on doping concentration became, however, inversed, if the doping concentration was higher than $0.03 \%$.

The role of the nanocomposite layer in the PLED device was to smooth the hole-injection progress. As demonstrated in the hole-only devices previously, because of the graphene doping, the conductivity of composite layer was apparently enhanced, and, the more the doping concentration, the higher the conductivity enhancement. When the doping concentration was not high, the enhancement of both efficiencies with the increase of doping concentration was attributable to the improvement of hole injection in the composite layer that led to more balanced holes and electrons combination in the emitting layer and thereby gaining higher overall efficiency. If the doping concentration kept increased, the nanocomposite layer's conductivity continued to be enhanced; more holes were injected and moved in a faster speed. The balance of hole-electron combination was altered, and, in addition, the location of carrier combination in the emitting layer was changed, toward to cathode. Thus, the efficiency was reduced. For the present study, the optimal doping concentration was around $0.03 \%$ where the luminous efficiency and luminous power efficiency were increased to $2.09 \mathrm{~cd} / \mathrm{A}$ and $0.79 \mathrm{~lm} / \mathrm{W}$, respectively, which were correspondingly 0.46 and 0.36 times of enhancement compared to the one without doping.

More complete electrical characteristics of the PLED device are listed in Table 1. It can be seen that, in addition to the reachable maximum luminance, current and power efficiencies, the turn-on voltage, and $V_{T}$ (the driving voltage as the luminance at $1 \mathrm{~cd} / \mathrm{m}^{2}$ ) could be improved by the graphene doping. The $V_{T}$ was $3.12 \mathrm{~V}$ for the undoped device, and its value was decreased with the increase of graphene concentration. It reached a lowest value of $2.6 \mathrm{~V}$ at a concentration of $0.05 \%$. When the doped concentration was higher than $0.05 \%$, the turn-on voltage was increased again. The variation in device's turn-on voltage is usually affected by the work functions of thin films $[9,25]$.

The work functions of ITO, PEDOT:PSS, and graphene are 5.1, 5.17, and $4.9 \mathrm{eV}$, respectively. These values and the HOMOs of composite layers are listed in the energy diagram shown in Figure 6. The energy barrier between ITO and PEDOT:PSS was $0.07 \mathrm{eV}$. The work function of graphene is $4.9 \mathrm{eV}$ which is lower than those of ITO and PEDOT:PSS. Thus, with the introduction of an appropriate amount of graphene in the PEDOT:PSS, the work function of this nanocomposite layer was reduced and the energy barrier was also lowered that facilitated the injection of holes and led to the reduction of the turn-on voltage. When the concentration was over than $0.05 \%$, the work functions of the composite films were lower than that of ITO. A Schottky barrier was formed at the contact which hindered hole injection and led to an increase of the turn-on voltage again. 


\section{Conclusions}

In conclusion, the PEDOT:PSS/graphene nanocomposite thin film, PEDOT:PSS doped with graphenes, was fabricated and its application as a hole-injection layer in PLEDs was studied. Graphene oxides were first synthesized by Hummers' method and then mixed in the PEDOT:PSS solution at several specific amounts. The PEDOT:PSS/graphene nanocomposite with particular graphene doping concentration was obtained by thermally reducing the graphene oxides into graphenes. The effect of doping concentration on the device performance was systematically examined, and the mechanism of characteristic modifications was discussed. The doping of graphene can effectively enhance the conductivity in the hole-injection layer and reduce the turn-on voltage that made the progress of hole injection more smoothly and led to a higher overall device efficiency. Results showed, compared with the case of using undoped hole-injection layer, that the maximum luminance could be enhanced up to 1.66 times while the efficiencies of luminous and luminous power were increased $46 \%$ and $36 \%$, respectively. Basically, the hole-injection ability was increased with the increase of doping concentration. However, if the doping concentration was too high, the device performance was reduced with the success increase of doping concentration. The optimal doping concentration was from the case using $0.03 \mathrm{wt} \%$ of graphene oxide.

\section{Acknowledgment}

Finical support from the National Science Foundation of Taiwan under Grant numbers NSC97-2221-E-194-058-MY3 and NSC99-2627-E-194-001 is greatly appreciated.

\section{References}

[1] S. A. Carter, J. C. Scott, and P. J. Brock, "Enhanced luminance in polymer composite light emitting devices," Applied Physics Letters, vol. 71, no. 9, pp. 1145-1147, 1997.

[2] N. N. Dinh, L. H. Chi, T. T. C. Thuy, T. Q. Trung, and V. $\mathrm{V}$. Truong, "Enhancement of current-voltage characteristics of multilayer organic light emitting diodes by using nanostructured composite films," Journal of Applied Physics, vol. 105, no. 9, Article ID 093518, 2009.

[3] J. Y. Kim, M. Kim, H. Kim, J. Joo, and J. H. Choi, "Electrical and optical studies of organic light emitting devices using SWCNTs-polymer nanocomposites," Optical Materials, vol. 21, no. 1-3, pp. 147-151, 2002.

[4] L. Qian, Y. Zheng, K. R. Choudhury et al., "Electroluminescence from light-emitting polymer/ZnO nanoparticle heterojunctions at sub-bandgap voltages," Nano Today, vol. 5, no. 5, pp. 384-389, 2010.

[5] I. Singh, D. Madhwal, A. Verma et al., "Enhanced luminance of MEH-PPV based PLEDs using single walled carbon nanotube composite as an electron transporting layer," Journal of Luminescence, vol. 130, no. 11, pp. 2157-2160, 2010.

[6] B. Riedel, J. Hauss, M. Aichholz, A. Gall, U. Lemmer, and M. Gerken, "Polymer light emitting diodes containing nanoparticle clusters for improved efficiency," Organic Electronics, vol. 11, no. 7, pp. 1172-1175, 2010.
[7] Z. B. Deng, X. M. Ding, S. T. Lee, and W. A. Gambling, "Enhanced brightness and efficiency in organic electroluminescent devices using $\mathrm{SiO}_{2}$ buffer layers," Applied Physics Letters, vol. 74, no. 15, pp. 2227-2229, 1999.

[8] I. M. Chan and F. C. Hong, "Improved performance of the single-layer and double-layer organic light emitting diodes by nickel oxide coated indium tin oxide anode," Thin Solid Films, vol. 450, no. 2, pp. 304-311, 2004.

[9] A. Gyoutoku, S. Hara, T. Komatsu, M. Shirinashihama, H. Iwanaga, and K. Sakanoue, "An organic electroluminescent dot-matrix display using carbon underlayer," Synthetic Metals, vol. 91, no. 1-3, pp. 73-75, 1997.

[10] Y. Shen, D. B. Jacobs, G. G. Malliaras, G. Koley, M. G. Spencer, and A. Ioannidis, "Modification of indium tin oxide for improved hole injection in organic light emitting diodes," Advanced Materials, vol. 13, no. 16, pp. 1234-1238, 2001.

[11] Y. Yang and A. J. Heeger, "Polyaniline as a transparent electrode for polymer light-emitting diodes: lower operating voltage and higher efficiency," Applied Physics Letters, vol. 64, no. 10, pp. 1245-1247, 1994.

[12] Y. Cao, G. Yu, C. Zhang, R. Menon, and A. J. Heeger, "Polymer light-emitting diodes with polyethylene dioxythiophenepolystyrene sulfonate as the transparent anode," Synthetic Metals, vol. 87, no. 2, pp. 171-174, 1997.

[13] A. Elschner, F. Bruder, H. W. Heuer et al., "PEDT/PSS for efficient hole-injection in hybrid organic light-emitting diodes," Synthetic Metals, vol. 111-112, pp. 139-143, 2000.

[14] H. S. Woo, R. Czerw, S. Webster, D. L. Carroll, J. W. Park, and J. H. Lee, "Organic light emitting diodes fabricated with single wall carbon nanotubes dispersed in a hole conducting buffer: the role of carbon nanotubes in a hole conducting polymer," Synthetic Metals, vol. 116, no. 1-3, pp. 369-372, 2001.

[15] G. F. Wang, X. M. Tao, and R. X. Wang, "Fabrication and characterization of OLEDs using PEDOT:PSS and MWCNT nanocomposites," Composites Science and Technology, vol. 68, no. 14, pp. 2837-2841, 2008.

[16] M. Shao, M. P. Garrett, X. Xu, I. N. Ivanov, S. S. Wong, and $\mathrm{B}$. $\mathrm{Hu}$, "Effects of single walled carbon nanotubes on the electroluminescent performance of organic light-emitting diodes," Organic Electronics, vol. 12, no. 6, pp. 1098-1102, 2011.

[17] Z. Liu, D. He, Y. Wang, H. Wu, and J. Wang, "Effect of SPFGraphene dopant in MEH-PPV organic light-emitting devices," Synthetic Metals, vol. 160, no. 13-14, pp. 1587-1589, 2010.

[18] J. H. Hwang, K. S. Lee, H. S. Bang et al., "Enhancement of the hole injection current in polymer light-emitting devices fabricated utilizing a graphene layer," Journal of the Electrochemical Society, vol. 158, no. 9, pp. J273-J275, 2011.

[19] H. Chang, G. Wang, A. Yang et al., "A transparent, flexible, low-temperature, and solution-processible graphene composite electrode," Advanced Functional Materials, vol. 20, no. 17, pp. 2893-2902, 2010.

[20] Z. Zhong, Y. Dai, D. Ma, and Z. Y. Wang, "Facile synthesis of organo-soluble surface-grafted all-single-layer graphene oxide as hole-injecting buffer material in organic light-emitting diodes," Journal of Materials Chemistry, vol. 21, no. 16, pp. 6040-6045, 2011.

[21] W. S. Hummers Jr. and R. E. Offeman, "Preparation of graphitic oxide," Journal of the American Chemical Society, vol. 80, no. 6, p. 1339, 1958.

[22] S. H. Huh, "Thermal reduction of graphene oxide," in Physics and Applications of Graphene-Experiments, S. Mikhailov, Ed., chapter 5, InTech, 2011. 
[23] R. Cervini, X.-C. Li, G. W. C. Spencer, A. B. Holmes, S. C. Moratti, and R. H. Friend, "Electrochemical and optical studies of PPV derivatives and poly(aromatic oxadiazoles)," Synthetic Metals, vol. 84, no. 1-3, pp. 359-360, 1997.

[24] N. G. Connelly and W. E. Geiger, "Chemical redox agents for organometallic chemistry," Chemical Reviews, vol. 96, no. 2, pp. 877-910, 1996.

[25] C. C. Oey, A. B. Djurixic, C. Y. Kwong et al., "Nanocomposite hole injection layer for organic device applications," Thin Solid Films, vol. 492, no. 1-2, pp. 253-258, 2005. 

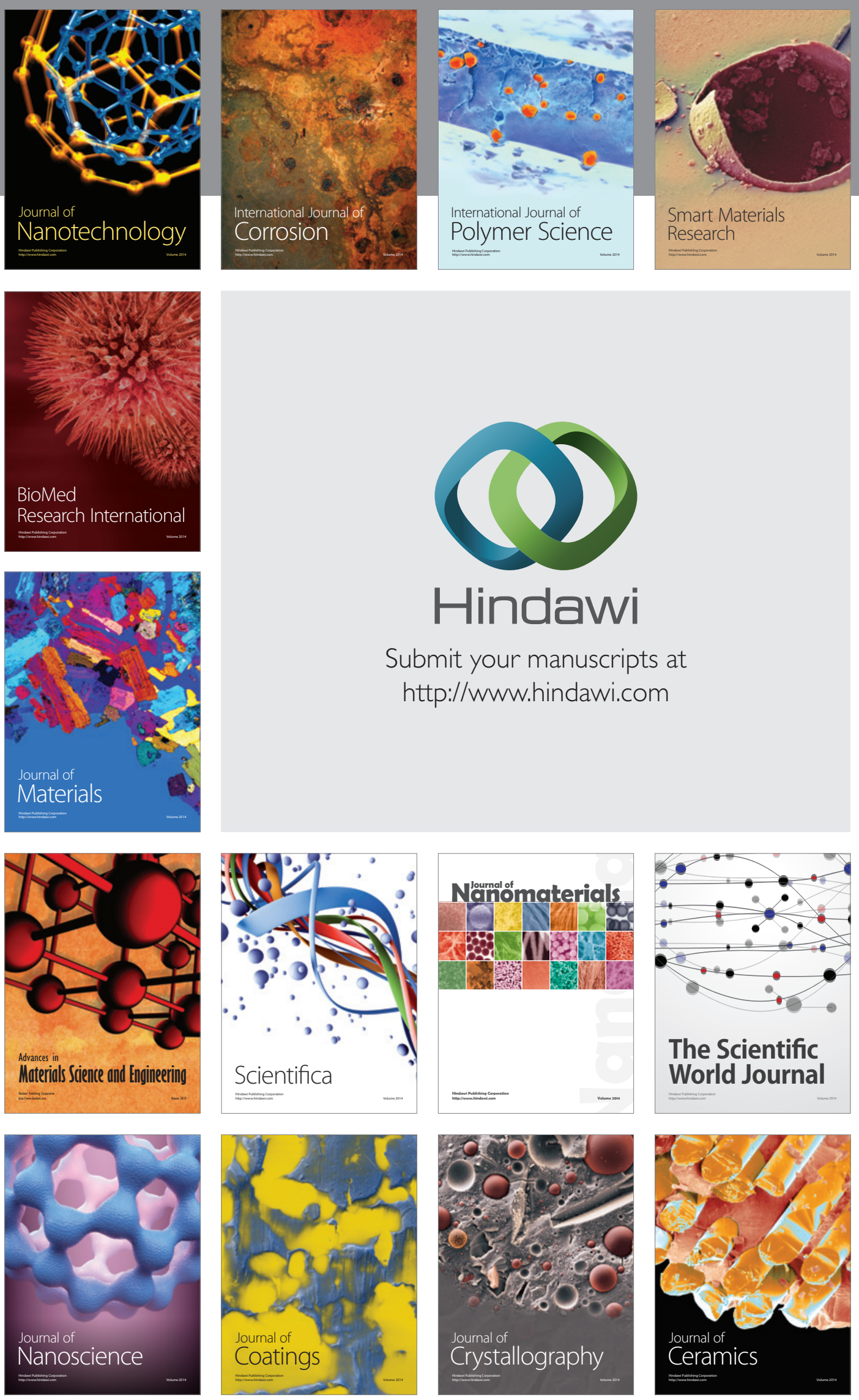

The Scientific World Journal

Submit your manuscripts at

http://www.hindawi.com

\section{World Journal}

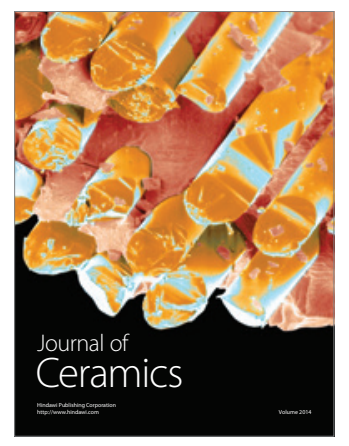

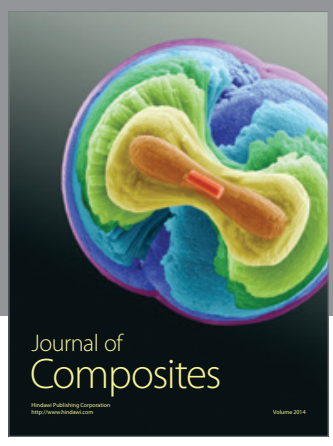
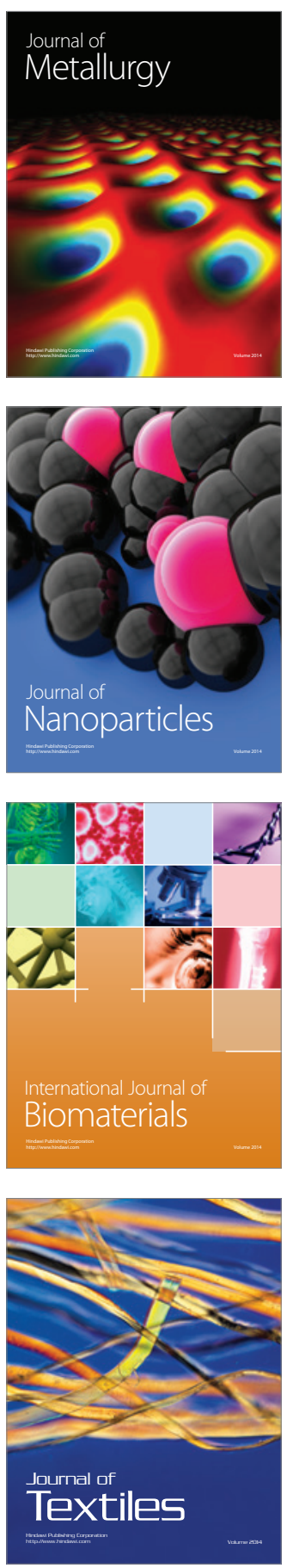